\title{
Superfluidity of a spin-imbalanced Fermi gas in a three-dimensional optical lattice
}

\author{
R. Mendoza \\ Posgrado en Ciencias Físicas, UNAM; Instituto de Física, UNAM \\ Mauricio Fortes and M. A. Solís \\ Instituto de Física, UNAM, Apdo. Postal 20-364, 01000 México D.F., México \\ Zlatko Koinov \\ Department of Physics and Astronomy, University of Texas at San Antonio, San Antonio, Texas 78249, USA
}

\begin{abstract}
We study fermion pairing in a population-imbalanced mixture of ${ }^{6} \mathrm{Li}$ atomic gas loaded in a three-dimensional lattice at very low temperatures. Using the number equation for each population, the gap equation and the equation for the Helmholtz free energy, we determine the gap, chemical potentials and pair-momentum as functions of polarization. These parameters define the stability regions for: a Fulde-Ferrell-Larkin-Ovchinnikov phase; a phase separation region where BCS and normal phases coexist; a Sarma phase when the pair-momentum vanishes, and the transition to the normal phase when the gap disappears. The collective-mode energies are then calculated using a Bethe-Salpeter approach in the generalized random phase approximation assuming that the system is well described by the single-band Hubbard model. A novel result is that this fermionic gas has a superfluid phase revealed by rotonlike minima in the asymmetric collective-mode energy spectrum.
\end{abstract}

PACS numbers: 67.85.-d, 03.75.Ss, 71.10.Pm, 73.20.Mf

Keywords: Superfluidity, Roton Polarized Fermi gas, Bethe-Salpeter

\section{INTRODUCTION}

The ability to use optical lattices to study the properties of ultracold atoms provides a testing model to simulate different strongly-correlated Fermi systems. Optical lattices are also tailored-made to study the effects of dimensionality on correlated Fermi systems as the former are created by standing laser waves in one, two or three dimensions $\frac{1}{*}$. Since the frequency and intensity of the lasers can be tuned up to specific values, the properties of ultracold Fermi or Bose systems loaded onto these lattices can be studied with impressive detail 2 . In addition, when the atoms are near a Feshbach resonance their interaction can be finely tuned to explore the crossover from the weakly-interacting Bardeen-CooperSchrieffer (BCS) regime characterized by Cooper pair formation to the strongly-interacting regime where the formation of molecular pairs with zero spin can undergo a Bose-Einstein condensation (BEC) at sufficiently low temperatures ${ }^{3}$. Although most experimental and theoretical models of correlated Fermi systems have dealt with balanced populations of spin states, more recently $\frac{4,5}{.5}$ the ability to manipulate ultracold atomic clouds has motivated the interest to study systems when the mixture of two hyperfine states in, for example, an atomic Fermi gas is not balanced. In this case, the two Fermi surfaces are no longer aligned and the lowest energy pairs have non-zero total momenta. Such phases were first studied by Fulde and Ferrell $(\mathrm{FF})^{6}$, who used an order parameter that varies as a single plane wave, and by Larkin and Ovchinnikov (LO $)^{7}$, who suggested that the order parameter is a superposition of two plane waves.

Although the FF and LO phases (presently referred as FFLO) were introduced quite a long time ago, they are still of very high interest because the question whether the superconductivity/superfluidity can survive in 3D polarized systems remains experimentally unanswered. In the FFLO phase, Cooper pairing occurs between a fermion with momentum $\boldsymbol{k}+\boldsymbol{q}$ and spin $\uparrow$ and a fermion with momentum $-\boldsymbol{k}+\boldsymbol{q}$, and spin $\downarrow$. As a result, the total pair momentum is $2 \boldsymbol{q}$ and the order parameter becomes spatially dependent as proposed by Larkin and Ovchinnikov ${ }^{\underline{7}}$. The mean-field treatment of the FFLO phase in a variety of systems, such as superconductors with Zeeman splitting and heavy-fermion superconductors ${ }^{8}$, atomic Fermi gases with population imbalance loaded in optical lattices $\underline{-11}$ and harmonic traps $\frac{12}{2}$, and dense quark matter $\underline{13}$ shows that the FFLO phase competes with a number of other phases, such as the Sarma $(\boldsymbol{q}=0)$ states $\frac{14}{}$, but in some regions of momentum space the FFLO phase is more stable as it provides the minimum of the mean-field expression of the Helmholtz free energy.

In this paper we calculate the polarization dependence of the gap, chemical potentials and pair-momentum as well as the collective excitations of an imbalanced mixture of two hyperfine states $|\uparrow\rangle$ and $|\downarrow\rangle$ of a ${ }^{6} \mathrm{Li}$ atomic Fermi gas under an attractive contact interaction loaded into a cubic optical lattice.

In Section II we summarize the properties of the Hubbard Hamiltonian used here to model a two-component Fermi gas in a lattice produced by standing waves of three pairs of counter-propagating laser beams. Section III is devoted to the calculation of the thermodynamic potential of the system. When the number of particles is fixed, the Helmholtz free energy is obtained as a function of the order parameter and total pair momentum. We also analyze the extent of the phase separation region determined 
by the minimal free energy of a normal and a BCS mixture. The polarization vs temperature phase diagram is calculated and compared with previous results for a $2 \mathrm{D}$ system ${ }^{15}$. In Section IV we derive a Bethe-Salpeter equation for two-body amplitudes assuming a generalized random-phase approximation. The collective excitations are obtained via the vanishing of a secular $4 \times 4$ determinant. Finally, our conclusions are presented in Section V.

\section{HUBBARD MODEL IN A CUBIC OPTICAL LATTICE}

The Hamiltonian of a two-component Fermi gas under an attractive contact interaction $v\left(\boldsymbol{r}-\boldsymbol{r}^{\prime}\right)=v_{0} \delta\left(\boldsymbol{r}-\boldsymbol{r}^{\prime}\right)$ is given by

$$
\begin{gathered}
H=\sum_{\sigma} \int d \boldsymbol{r} \hat{\Psi}_{\sigma}^{\dagger}(\boldsymbol{r})\left[-\frac{\hbar^{2} \nabla^{2}}{2 m}+V_{\sigma}(\boldsymbol{r})-\mu_{\sigma}\right] \hat{\Psi}_{\sigma}(\boldsymbol{r}) \\
+v_{0} \iint d \boldsymbol{r} d \boldsymbol{r}^{\prime} \hat{\Psi}_{\sigma_{1}}^{\dagger}(\boldsymbol{r}) \hat{\Psi}_{\sigma_{2}}^{\dagger}\left(\boldsymbol{r}^{\prime}\right) \delta\left(\boldsymbol{r}-\boldsymbol{r}^{\prime}\right) \hat{\Psi}_{\sigma_{2}}\left(\boldsymbol{r}^{\prime}\right) \hat{\Psi}_{\sigma_{1}}(\boldsymbol{r}),(1)
\end{gathered}
$$

where $\hat{\Psi}_{\sigma}^{\dagger}(\boldsymbol{r})$ and $\hat{\Psi}_{\sigma}(\boldsymbol{r})$ are fermionic creation and annihilation field operators of component $\sigma$, respectively; $\mu_{\sigma}$ is the chemical potential for each component $\mid \uparrow>$ or $|\downarrow\rangle$, and the lattice periodic potential is

$$
V_{\sigma}(\boldsymbol{r})=\gamma_{\sigma, x} \sin ^{2} k x+\gamma_{\sigma, y} \sin ^{2} k y+\gamma_{\sigma, z} \sin ^{2} k z,
$$

where $k=\pi / a$ with $a=\lambda / 2$, the lattice constant and $\lambda$ is the laser wavelength.

We will assume that the optical-lattice potential strengths $\gamma_{\sigma, \nu}(\nu=x, y$ or $z)$ are sufficiently deep to consider that lattice-site tunneling occurs only between nearest neighbors. Then, the field operators can be expanded as

$$
\hat{\Psi}_{\sigma}(\boldsymbol{r})=\sum_{i} \psi_{i, \sigma}(\boldsymbol{r}) \hat{c}_{i, \sigma}
$$

where $\psi_{i, \sigma}(\boldsymbol{r})$ are one-particle wave functions localized at site $i$, and the Fermi operator $\hat{c}_{i, \sigma}^{\dagger}\left(\hat{c}_{i, \sigma}\right)$ creates (destroys) an atom in site $i$. Under these assumptions, the Hamiltonian in (1) reduces to the single-band attractive Hubbard model,

$$
\begin{aligned}
H= & -J_{x} \sum_{\langle i, j\rangle_{x}, \sigma} \hat{c}_{i, \sigma}^{\dagger} \hat{c}_{j, \sigma}-J_{y} \sum_{\langle i, j\rangle_{y}, \sigma} \hat{c}_{i, \sigma}^{\dagger} \hat{c}_{j, \sigma} \\
& -J_{z} \sum_{\langle i, j\rangle_{z}, \sigma} \hat{c}_{i, \sigma}^{\dagger} \hat{c}_{j, \sigma}-\sum_{i}\left(\mu_{\uparrow}^{\dagger} \hat{c}_{i, \uparrow}^{\dagger} \hat{c}_{i, \uparrow}+\mu_{\downarrow} \hat{c}_{i, \downarrow}^{\dagger} \hat{c}_{i, \downarrow}\right) \\
& +U \sum_{i} \hat{c}_{i, \uparrow}^{\dagger} \hat{c}_{i, \downarrow}^{\dagger} \hat{c}_{i, \downarrow} \hat{c}_{i, \uparrow},
\end{aligned}
$$

where $J_{\nu}$ is the tunneling strength of the atoms between nearest-neighbor sites in the $\nu$-direction and $U$ is the onsite attractive interaction strength. On the BCS side, the
Hubbard parameter $U$ is negative, but in what follows $U$ denotes its absolute value and is given by

$$
U=v_{0} \int d \boldsymbol{r}\left|\psi_{i, \uparrow}(\boldsymbol{r})\right|^{2}\left|\psi_{i, \downarrow}(\boldsymbol{r})\right|^{2} .
$$

We assume a system with a total number of atoms $M=M_{\uparrow}+M_{\downarrow}$ distributed along $N$ sites of the opticallattice potential (2). In the mean-field approximation, the pair interaction term in Eq. (3) is replaced by

$$
\begin{aligned}
& U \sum_{i} \hat{c}_{i, \uparrow}^{\dagger} \hat{c}_{i, \downarrow}^{\dagger} \hat{c}_{i, \downarrow} \hat{c}_{i, \uparrow} \simeq U \sum_{i}\left(\left\langle\hat{c}_{i, \uparrow}^{\dagger} \hat{c}_{i, \downarrow}^{\dagger}\right\rangle \hat{c}_{i, \downarrow} \hat{c}_{i, \uparrow}\right. \\
& \left.+\hat{c}_{i, \uparrow}^{\dagger} \hat{c}_{i, \downarrow}^{\dagger}\left\langle\hat{c}_{i, \downarrow} \hat{c}_{i, \uparrow}\right\rangle-\left\langle\hat{c}_{i, \uparrow}^{\dagger} \hat{c}_{i, \downarrow}^{\dagger}\right\rangle\left\langle\hat{c}_{i, \downarrow} \hat{c}_{i, \uparrow}\right\rangle\right) .
\end{aligned}
$$

The order parameter $\Delta_{i}=U\left\langle\hat{c}_{i, \downarrow} \hat{c}_{i, \uparrow}\right\rangle$ of the FFLO states is assumed to vary as a single plane wave, $\Delta_{i}=$ $\Delta \exp \left(2 \imath \boldsymbol{q} \cdot \boldsymbol{r}_{i}\right)$, where $\boldsymbol{q}$ is the pair center-of-mass momentum and $\boldsymbol{r}_{i}$ the coordinate of site $i$. These states are expected to occur on the BCS side of a Feshbach resonance where the effective attractive interaction between fermion atoms leads to BCS-type pairing. The tight-binding lattice dispersion energy is $\xi_{\uparrow, \downarrow}(\boldsymbol{k})=$ $2 J\left(1-\sum_{\nu} \cos k_{\nu} a\right)-\mu_{\uparrow, \downarrow}$. In our calculations we use $\lambda=1030 \mathrm{~nm}$ and equal tunneling strengths $J_{\nu}=J$ to obtain the following mean-field Hamiltonian,

$$
\begin{aligned}
H & =\frac{1}{N} \sum_{\boldsymbol{k}}\left[\xi_{\uparrow}(\boldsymbol{k}) \hat{c}_{\boldsymbol{k}, \uparrow}^{\dagger} \hat{c}_{\boldsymbol{k}, \uparrow}+\xi_{\downarrow}(\boldsymbol{k}) \hat{c}_{\boldsymbol{k}, \downarrow}^{\dagger} \hat{c}_{\boldsymbol{k}, \downarrow}\right. \\
& \left.+\Delta \hat{c}_{\boldsymbol{k}+\boldsymbol{q}, \uparrow}^{\dagger} \hat{c}_{-\boldsymbol{k}+\boldsymbol{q}, \downarrow}^{\dagger}+\Delta^{*} \hat{c}_{-\boldsymbol{k}+\boldsymbol{q}, \downarrow} \hat{c}_{\boldsymbol{k}+\boldsymbol{q}, \uparrow}+\frac{|\Delta|^{2}}{U}\right],
\end{aligned}
$$

which can be diagonalized using a Bogoliubov transformation ${ }^{9}$,

$$
\left(\begin{array}{c}
\hat{c}_{\boldsymbol{k}+\boldsymbol{q}, \uparrow} \\
\hat{c}_{-\boldsymbol{k}+\boldsymbol{q}, \downarrow}^{\dagger}
\end{array}\right)=\left(\begin{array}{cc}
u_{\boldsymbol{q}}(\boldsymbol{k}) & v_{\boldsymbol{q}}(\boldsymbol{k}) \\
-v_{\boldsymbol{q}}(\boldsymbol{k}) & u_{\boldsymbol{q}}(\boldsymbol{k})
\end{array}\right)\left(\begin{array}{c}
\hat{d}_{\boldsymbol{k}, \boldsymbol{q}, \uparrow} \\
\hat{d}_{-\boldsymbol{k}, \boldsymbol{q}, \downarrow}^{\dagger}
\end{array}\right) .
$$

The coefficients $u_{\boldsymbol{q}}, v_{\boldsymbol{q}}$ are given by

$$
u_{\boldsymbol{q}}(\boldsymbol{k})=\sqrt{\frac{1}{2}\left[1+\frac{\chi_{\boldsymbol{q}}(\boldsymbol{k})}{E_{\boldsymbol{q}}(\boldsymbol{k})}\right]}, \quad v_{\boldsymbol{q}}(\boldsymbol{k})=\sqrt{\frac{1}{2}\left[1-\frac{\chi_{\boldsymbol{q}}(\boldsymbol{k})}{E_{\boldsymbol{q}}(\boldsymbol{k})}\right]},
$$

where

$$
\begin{aligned}
& \chi_{\boldsymbol{q}}(\boldsymbol{k})=\frac{1}{2}\left[\xi_{\uparrow}(\boldsymbol{k}+\boldsymbol{q})+\xi_{\downarrow}(\boldsymbol{q}-\boldsymbol{k})\right], \\
& E_{\boldsymbol{q}}(\boldsymbol{k})=\sqrt{\chi_{\boldsymbol{q}}^{2}(\boldsymbol{k})+\Delta^{2}}
\end{aligned}
$$

\section{THERMODYNAMIC POTENTIAL AND PHASE DIAGRAMS}

In the mean-field approximation, the momentumspace, single-particle Green function is a $2 \times 2$ matrix given by

$$
\widehat{G}=\left(\begin{array}{cc}
G_{\boldsymbol{q}}^{\uparrow \uparrow} & G_{\boldsymbol{q}}^{\uparrow \downarrow} \\
G_{\boldsymbol{q}}^{\downarrow \uparrow} & G_{\boldsymbol{q}}^{\downarrow \downarrow}
\end{array}\right)
$$


where

$$
\begin{array}{r}
G_{\boldsymbol{q}}^{\uparrow \uparrow}\left(\boldsymbol{k}, \imath \omega_{m}\right)=\frac{u_{\boldsymbol{q}}(\boldsymbol{k})^{2}}{\imath \hbar \omega_{m}-\omega_{+}(\boldsymbol{k}, \boldsymbol{q})}+\frac{v_{\boldsymbol{q}}(\boldsymbol{k})^{2}}{\imath \hbar \omega_{m}+\omega_{-}(\boldsymbol{k}, \boldsymbol{q})}, \\
G_{\boldsymbol{q}}^{\downarrow \downarrow}\left(\boldsymbol{k}, \imath \omega_{m}\right)=\frac{v_{\boldsymbol{q}}(\boldsymbol{k})^{2}}{\imath \hbar \omega_{m}-\omega_{+}(\boldsymbol{k}, \boldsymbol{q})}+\frac{u_{\boldsymbol{q}}(\boldsymbol{k})^{2}}{\imath \hbar \omega_{m}+\omega_{-}(\boldsymbol{k}, \boldsymbol{q})}, \\
G_{\boldsymbol{q}}^{\uparrow \downarrow}\left(\boldsymbol{k}, \imath \omega_{m}\right)=G_{\boldsymbol{q}}^{\downarrow \uparrow}\left(\boldsymbol{k}, \imath \omega_{m}\right)=u_{\boldsymbol{q}}(\boldsymbol{k}) v_{\boldsymbol{q}}(\boldsymbol{k}) \times \\
{\left[\frac{1}{\imath \hbar \omega_{m}-\omega_{+}(\boldsymbol{k}, \boldsymbol{q})}-\frac{1}{\imath \hbar \omega_{m}+\omega_{-}(\boldsymbol{k}, \boldsymbol{q})}\right] .}
\end{array}
$$

The Matsubara frequencies are $\omega_{m}=\pi k_{B} T(2 m+1) / \hbar$ with $m=0, \pm 1, \pm 2, \ldots ; T$ is the temperature, and $k_{B}$ the Boltzmann constant. The one-particle excitations in a mean-field approximation $\omega_{ \pm}$are coherent combinations of electronlike $\omega_{+}(\boldsymbol{k}, \boldsymbol{q})=E_{\boldsymbol{q}}(\boldsymbol{k})+\eta_{\boldsymbol{q}}(\boldsymbol{k})$ and holelike $\omega_{-}(\boldsymbol{k}, \boldsymbol{q})=E_{\boldsymbol{q}}(\boldsymbol{k})-\eta_{\boldsymbol{q}}(\boldsymbol{k})$ excitations, where $\eta_{\boldsymbol{q}}(\boldsymbol{k})=\frac{1}{2}\left[\xi_{\uparrow}(\boldsymbol{k}+\boldsymbol{q})-\xi_{\downarrow}(\boldsymbol{q}-\boldsymbol{k})\right]$. The thermodynamic potential at temperature $T$ in a mean field approximation can be evaluated from the grand canonical partition function $Z$ of an ensemble of quasiparticles with energy $\omega_{ \pm}(\boldsymbol{k}, \boldsymbol{q})$ given by ${ }^{9}$

$$
\begin{array}{r}
Z=\prod_{\boldsymbol{k}}\left(1+e^{-\beta \omega_{+}(\boldsymbol{k}, \boldsymbol{q}) / N}\right)\left(1+e^{\beta \omega_{-}(\boldsymbol{k}, \boldsymbol{q}) / N}\right) \times \\
e^{-\frac{\beta}{N}\left(\chi_{\boldsymbol{q}}(\boldsymbol{k})+\frac{|\Delta|^{2}}{U}\right)},
\end{array}
$$

where $\beta=1 / k_{B} T$. The thermodynamic potential $\Omega=$ $-\frac{1}{\beta} \ln Z$ is therefore,

$$
\begin{gathered}
\Omega=\frac{1}{N} \sum_{\boldsymbol{k}}\left[\chi_{\boldsymbol{q}}(\boldsymbol{k})+\omega_{-}(\boldsymbol{k}, \boldsymbol{q})+\frac{\Delta^{2}}{U}\right] \\
-\frac{1}{\beta} \sum_{\boldsymbol{k}}\left[\ln \left(1+e^{-\beta \omega_{+}(\boldsymbol{k}, \boldsymbol{q})}\right)+\ln \left(1+e^{\beta \omega_{-}(\boldsymbol{k}, \boldsymbol{q})}\right)\right] .
\end{gathered}
$$

From (8), the parameter $\Delta=\frac{U}{N} \sum_{\boldsymbol{k}}\left\langle\hat{c}_{-\boldsymbol{k}+\boldsymbol{q}, \downarrow} \hat{c}_{\boldsymbol{k}+\boldsymbol{q}, \uparrow}\right\rangle$ satisfies the gap equation at zero temperature

$$
1=\frac{U}{N} \sum_{\boldsymbol{k}} \frac{1}{2 E_{\boldsymbol{q}}(\boldsymbol{k})} .
$$

If we consider an imbalanced system with fixed chemical potentials, $\mu_{\uparrow, \downarrow}$, the minima of $\Omega\left(\Delta, \boldsymbol{q}, \mu_{\uparrow}, \mu_{\downarrow}, T\right)$ with respect to $\Delta, \boldsymbol{q}, \mu_{\uparrow}, \mu_{\downarrow}$ define the possible stable phases of this system as a function of temperature. However, recent experiments, 16 deal with the more realistic situation in which the number of particles of each kind is fixed. In the latter case, the relevant thermodynamic potential is the Helmholtz free energy $F\left(\Delta, \boldsymbol{q}, f_{\uparrow}, f_{\downarrow}, T\right)=$ $\Omega+\mu_{\uparrow} f_{\uparrow}+\mu_{\downarrow} f_{\downarrow}$. Without loss of generality we set $\boldsymbol{q}=\left(q_{x}, 0,0\right)$, i.e., in the $x$-direction, and minimize the Helmholtz free energy $F\left(\Delta, q_{x}, f_{\uparrow}, f_{\downarrow}, T\right)$ with respect to $\mu_{\uparrow}, \mu_{\downarrow}, \Delta$ and $q_{x}$, where $f_{\uparrow, \downarrow} \equiv M_{\uparrow, \downarrow} / N$. As a result, we obtain a set of four equations, namely the number and gap equations, as well as the equation for $q_{x}$ :

$$
\begin{aligned}
& f_{\uparrow}=\frac{1}{N} \sum_{\boldsymbol{k}}\left[u_{\boldsymbol{q}}^{2}(\boldsymbol{k}) f\left(\omega_{+}(\boldsymbol{k}, \boldsymbol{q})\right)+v_{\boldsymbol{q}}^{2}(\boldsymbol{k}) f\left(-\omega_{-}(\boldsymbol{k}, \boldsymbol{q})\right)\right], \\
& f_{\downarrow}=\frac{1}{N} \sum_{\boldsymbol{k}}\left[u_{\boldsymbol{q}}^{2}(\boldsymbol{k}) f\left(\omega_{-}(\boldsymbol{k}, \boldsymbol{q})\right)+v_{\boldsymbol{q}}^{2}(\boldsymbol{k}) f\left(-\omega_{+}(\boldsymbol{k}, \boldsymbol{q})\right)\right], \\
& 1=\frac{U}{N} \sum_{\boldsymbol{k}} \frac{1-f\left(\omega_{-}(\boldsymbol{k}, \boldsymbol{q})\right)-f\left(\omega_{+}(\boldsymbol{k}, \boldsymbol{q})\right)}{2 E_{\boldsymbol{q}}(\boldsymbol{k})} \\
& 0=\frac{1}{N} \sum_{\boldsymbol{k}}\left\{\frac{\partial \eta_{\boldsymbol{q}}(\boldsymbol{k})}{\partial q_{x}}\left[f\left(\omega_{+}(\boldsymbol{k}, \boldsymbol{q})\right)-f\left(\omega_{-}(\boldsymbol{k}, \boldsymbol{q})\right)\right]+\frac{\partial \chi_{\boldsymbol{q}}(\boldsymbol{k})}{\partial q_{x}}\right. \\
& \left.\times\left[1-\frac{\chi_{\boldsymbol{q}}(\boldsymbol{k})}{E_{\boldsymbol{q}}(\boldsymbol{k})}\left[1-f\left(\omega_{+}(\boldsymbol{k}, \boldsymbol{q})\right)-f\left(\omega_{-}(\boldsymbol{k}, \boldsymbol{q})\right)\right]\right]\right\},(14)
\end{aligned}
$$

where $f\left(\omega_{ \pm}(\boldsymbol{k}, \boldsymbol{q})\right)=\left\langle\hat{d}_{-\boldsymbol{k}, \boldsymbol{q}, \uparrow}^{\dagger} \hat{d}_{\boldsymbol{k}, \boldsymbol{q}, \uparrow}\right\rangle$ is the Fermi distribution $\left[\exp \left(\beta \omega_{ \pm}(\boldsymbol{k}, \boldsymbol{q})\right)+1\right]^{-1}$.

The existence of mixed phases of normal state and superfluid has been reported in several analysis 10,17 . It arises when a fraction of the fermions are forming Cooper pairs in a BCS, Sarma or FFLO phase while the remaining (imbalanced) atoms are in the normal phase. Here, we only consider a configuration in which a fraction $(1-x)$ are in the BCS phase which requires equal numbers of $|\uparrow\rangle$ and $|\downarrow\rangle$ states with opposite momenta. The free energy in this mixed or phase separation (PS) state is

$$
F_{P S}=x F_{N}+(1-x) F_{B C S},
$$

where

$$
F_{B C S}=\Omega_{B C S}+\mu(1-x) \tilde{f},
$$

and $(1-x) \tilde{f}$ is the filling-factor fraction of fermions in the BCS state, and $\mu$ is the chemical potential with similar expressions for the filling-factor fraction in the normal phase given by

$$
F_{N}=\Omega_{N}+\mu_{\uparrow}\left(f_{\uparrow}-(1-x) \tilde{f}\right)+\mu_{\downarrow}\left(f_{\downarrow}-(1-x) \tilde{f}\right),
$$

where the thermodynamic potential in the normal phase is

$$
\Omega_{N}=-\frac{1}{\beta} \sum_{\mathbf{k}}\left\{\ln \left[\left(1+e^{-\beta \Omega_{\uparrow}}\right)\left(1+e^{-\beta \Omega_{\downarrow}}\right)\right]\right\} .
$$

The free energy is now also a function of $x, \tilde{f}$ and $\mu$, i.e., $F_{P S}=F_{P S}\left(x, \tilde{f}, \mu, \mu_{\uparrow}, \mu_{\downarrow}, \Delta\right)$. The minimum of $F_{P S}$ with respect to variations in the normal fraction $x$ or $\tilde{f}$ results in the following two additional relations

$$
\begin{aligned}
\tilde{f}\left(\mu_{\uparrow}+\mu_{\downarrow}\right) & =\Omega_{N}-\Omega_{B C S}+\mu_{\uparrow} f_{\uparrow}+\mu_{\downarrow} f_{\downarrow}, \\
\mu(1-x) & =x\left(\mu_{\uparrow}+\mu_{\downarrow}\right),
\end{aligned}
$$

which together with Eqs. (14) provide a system of six equations that define the equilibrium values of the thermodynamic variables. Since at a finite temperature the 


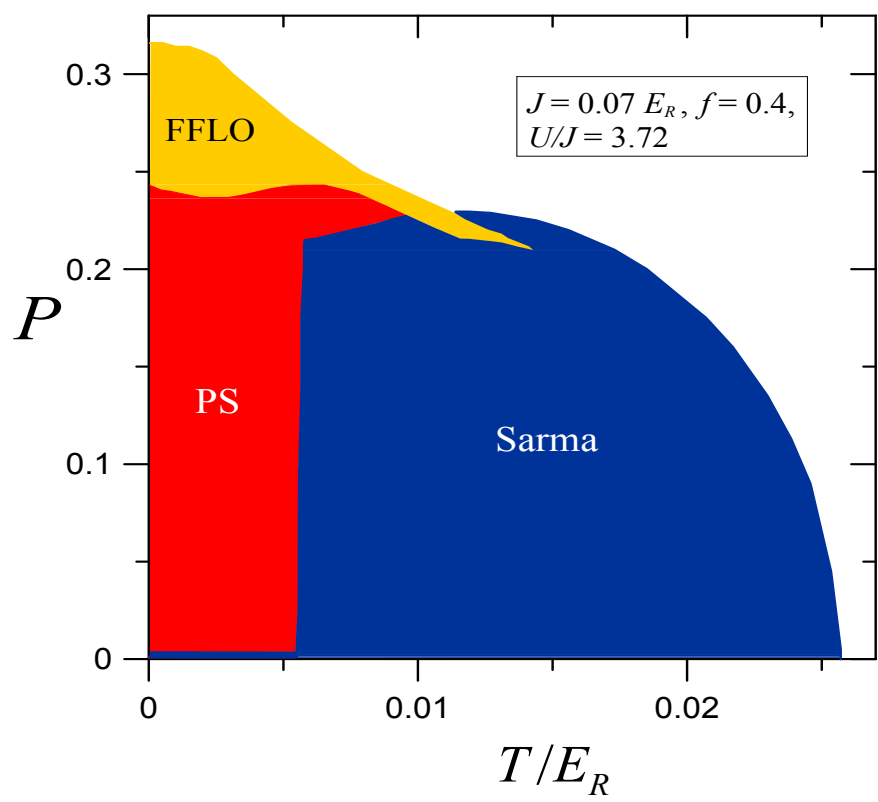

FIG. 1: (Color online). Phase diagram of a polarized ${ }^{6} \mathrm{Li}$ gas in a $3 \mathrm{D}$ optical lattice with $\lambda=1010 \mathrm{~nm}$ and filling factor $f=$ 0.4. FFLO (yellow), phase separation (red), Sarma (blue). The Hubbard parameters are $J=0.07 E_{R}$ and the attractive on-site attractive interaction is $U / J=3.72$.

FFLO, Sarma, PS and normal states compete with each other, we have calculated the regions in the $P$ vs $T$ plane that minimize the free energy. Here, $P$ is the polarization defined by

$$
P=\frac{f_{\uparrow}-f_{\downarrow}}{f_{\uparrow}+f_{\downarrow}}
$$

In Fig. 1 we exhibit the phase diagram of a threedimensional imbalanced system for a total filling factor $f=f_{\uparrow}+f_{\downarrow}=0.4, J=0.07 E_{R}$, and $U / J=3.72$, where $E_{R}=\hbar^{2}(2 \pi / \lambda)^{2} / 2 m$ is the recoil energy. We first choose these values for the parameters in order to compare with the results of reference $\mathrm{e}^{10}$. At low temperatures and polarization $P \geq 0.25$ the FFLO states are shown to be more stable than the Sarma phase, where the latter is characterized by $\boldsymbol{q}=0, \Delta \neq 0$ and $P \neq 0$. The stability phase region of the FFLO states extends to temperatures up to $k_{B} T / E_{R} \approx 0.015$ albeit over a narrower polarization interval compared to that obtained in ${ }^{10}$. This difference may be due to the definition of the phase separation phase given in Eq. (15). In the BCS phase, $\boldsymbol{q}=0$ and the number of particles of each species is the same, i.e., $P=0$. There is also the mixed phase region composed of normal and superfluid states where a fraction of the fluid is in the normal phase while the remaining fraction is in the BCS phase 17 .

In Fig. 2 we show the phase diagram for the same system but with a weaker attraction term $U / J=2.64$ and $f=0.4685$. This value for the on-site attraction coincides with our previous results 15 in $2 \mathrm{D}$ as we are interested in analyzing the effects of dimensionality on these systems. A decrease in $U$ and a slight increase in $J$ enhances the hopping between nearest neighboring sites. The overall effect is to expand the stability region of the FFLO phase in relation to the Sarma states compared to the phase diagram of Fig. 1, The largest polarization that the system can support before it becomes a normal fluid is $P=0.124$. In this case, the FFLO states lower the system free energy over quite a large phase region compared to the corresponding Sarma states at low temperatures. As the temperature increases, a sliver of Sarma states provides the minimum of the free energy. If the temperature is increased even further, the normal polarized Fermi gas becomes the energetically favored phase. Here, the phase-separation region and the FFLO phase dominate over the Sarma states.

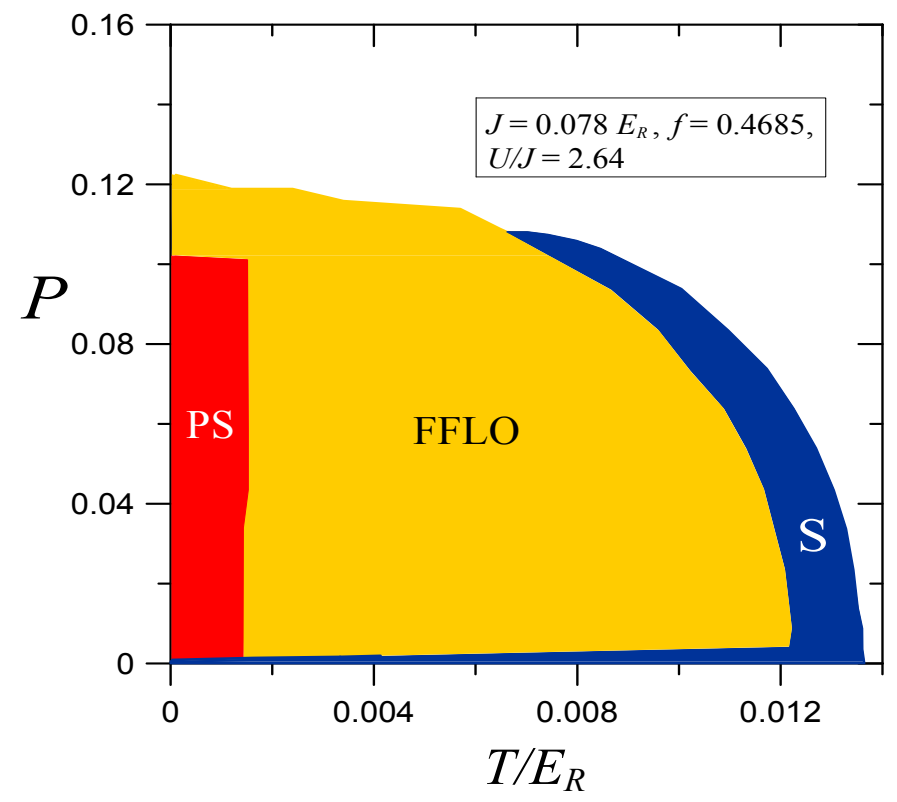

FIG. 2: (Color online). FFLO (yellow), phase separation (red) and Sarma (blue) phases of a polarized ${ }^{6} \mathrm{Li}$ gas in a $3 \mathrm{D}$ optical lattice with $\lambda=1030 \mathrm{~nm}$ and filling factor $f=0.4685$. The Hubbard parameters are $J=0.078 E_{R}$ and the attractive on-site attractive interaction is $U / J=2.64$.

In Figs. 3 and 4 we exhibit the variation with the polarization of the chemical potential of each species, the pair-momentum and the gap at a fixed temperature $k_{B} T=10^{-4} E_{R}$. Figure 3 shows the results for a $3 \mathrm{D}$ system where it remains as a FFLO superfluid up to $P \simeq 0.124$. At this value of the polarization the gap vanishes and therefore it enters a normal phase. In contrast, Fig. 4 shows the behavior of these quantities in a $2 \mathrm{D}$ system with the same parameters $U, J$ and $f$. It is interesting to note that even though the variation of $\mu_{\uparrow}, \mu_{\downarrow}, \Delta$ and $q_{x}$ follows the same trend as in the $3 \mathrm{D}$ case, the system remains a FFLO superfluid up to a somewhat higher value of the polarization, $P \simeq 0.18$ in the $2 \mathrm{D}$ regime. 


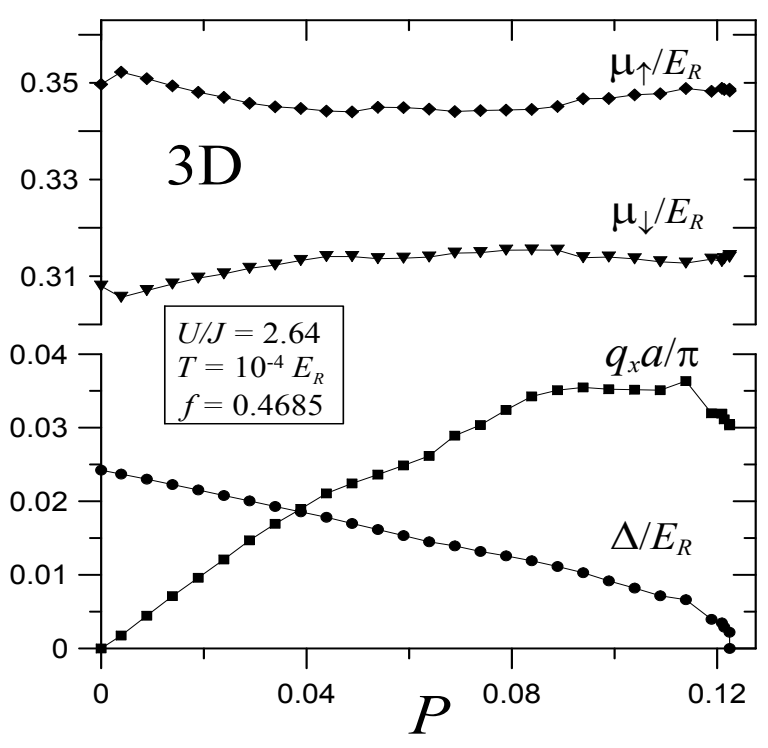

FIG. 3: Chemical potentials, pair momentum and gap for an imbalanced fermion gas loaded in a $3 \mathrm{D}$ optical lattice at $k_{B} T=10^{-4} E_{R}$

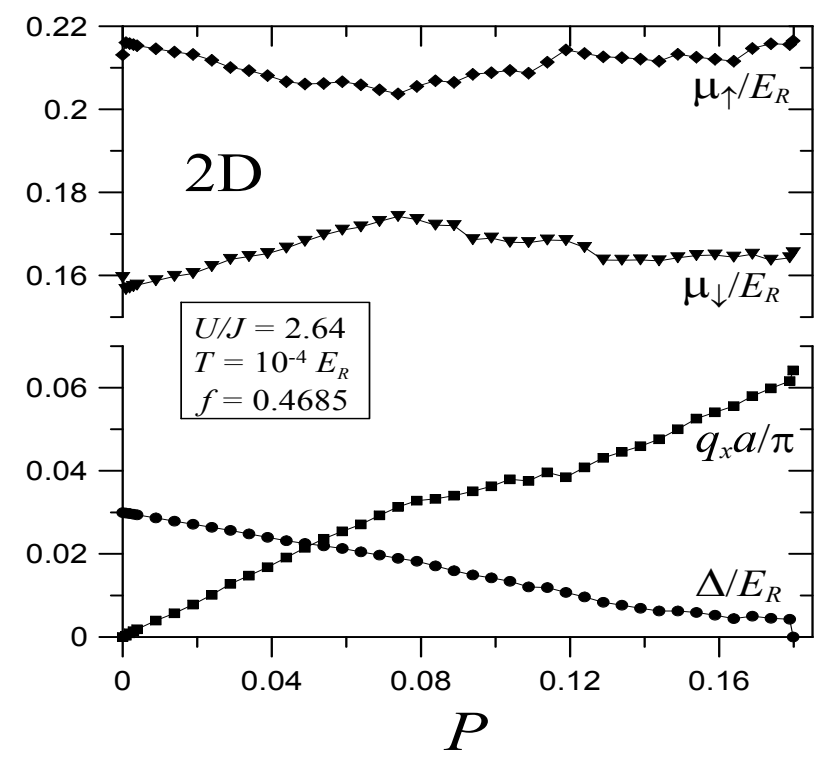

FIG. 4: Chemical potentials, pair momentum and gap for an imbalanced fermion gas loaded in a $2 \mathrm{D}$ optical lattice with $k_{B} T=10^{-4} E_{R}$.

\section{COLLECTIVE STATES}

Unlike the population-balanced systems, for which the spectrum of the collective excitations has been obtained by linearizing the Anderson-Rickayzen equations ${ }^{18}$, by the Kadanoff and Baym approach ${ }^{19}$ and by the BetheSalpeter (BS) formalism ${ }^{20}$, the FFLO collective modes have been studied in: (i) a $1 \mathrm{D}$ population-unbalanced trapped system 12 by using the linear response of the equilibrium system by supplementing the Bogoliubov- de Gennes (BdG) equations with a self-consistent random phase approximation; (ii) a 1D superconductor ${ }^{21}$ by transforming slow deformations of the order parameter into small corrections to the BdG Hamiltonian; and (iii) a cold-atom rotated system ${ }^{22}$ by locating the poles of the many-body scattering function. Here, we present a theory that goes beyond the mean-field approaches to find the spectrum of the collective excitations in the presence of FFLO phase by solving the BS equations for this spectrum in the general random phase approximation (GRPA) in a 3D optical lattice ${ }^{15}$.

The spectrum of the collective modes can be obtained from the poles of the two-particle Green's function $K(1,2 ; 3,4)$, where we use the compact notation $1=\left\{\sigma_{1}, \boldsymbol{r}_{1}, t_{1}\right\}, 2=\left\{\sigma_{2}, \boldsymbol{r}_{2}, t_{2}\right\}, \ldots$ with $\sigma_{i}$ denoting the spin variables, $\boldsymbol{r}_{i}$ the vector for lattice site $i$, and $t_{i}$, the time variable. $K$ satisfies the following Dyson equation:

$$
K=K_{0}+K_{0} I K
$$

where $K_{0}(1,2 ; 3,4)$ is the two-particle free propagator which is defined by a pair of fully dressed single-particle Green's function,

$$
K_{0}(1,2 ; 3,4)=G(1 ; 3) G(4 ; 2) .
$$

The interaction kernel $I$ is given by the functional derivatives of the mass operator $\Sigma(1 ; 2)=\Sigma_{D}(1 ; 2)+\Sigma_{E}(1 ; 2)$ obtained from the direct (or Fock) and exchange (or Hartree) parts, $I=\frac{\delta \Sigma}{\delta G}=\frac{\delta \Sigma_{D}}{\delta G}+\frac{\delta \Sigma_{E}}{\delta G}$. The Dyson equation for $G$ is

$$
\widehat{G}=G_{0}+G_{0} \Sigma \widehat{G}
$$

and therefore, the equation for the two-particle Green's function (21) must be solved self-consistently with (22). Since we are interested in the collective energy $\omega(\boldsymbol{Q})$ and momentum $Q$ excitations which are given by the poles of the two-particle fully dressed Green's function, we write the latter using the spectral representation

$$
\begin{gathered}
K(1,2 ; 3,4)=\sum_{\omega_{p}} e^{-i \omega_{p}\left(u_{1}-u_{3}\right)} \\
\times \frac{\Phi_{\boldsymbol{Q} ; \sigma_{1}, \sigma_{2}}\left(\boldsymbol{r}_{i_{1}}, \boldsymbol{r}_{i_{2}} ; u_{1}-u_{2}\right) \Phi_{\boldsymbol{Q}}^{*}\left(\boldsymbol{r}_{i_{3}}, \boldsymbol{r}_{i_{4}} ; u_{3}-u_{4}\right)}{i \omega_{p}-\omega(\boldsymbol{Q})},
\end{gathered}
$$

where $\Phi_{\boldsymbol{Q} ; \sigma_{1}, \sigma_{2}}\left(\boldsymbol{r}_{i_{1}}, \boldsymbol{r}_{i_{2}} ; u_{2}-u_{1}\right)$ are the BS amplitudes

$$
\begin{aligned}
\Phi_{\boldsymbol{Q} ; \sigma_{1}, \sigma_{2}}\left(\boldsymbol{r}_{i_{1}}, \boldsymbol{r}_{i_{2}} ; u_{2}-u_{1}\right)= & e^{i \boldsymbol{Q} \cdot\left(\boldsymbol{r}_{i_{1}}+\boldsymbol{r}_{i_{2}}\right) / 2} \times \\
& \phi_{\boldsymbol{Q} ; \sigma_{1}, \sigma_{2}}\left(\boldsymbol{r}_{i_{1}}-\boldsymbol{r}_{i_{2}} ; u_{1}-u_{2}\right) .
\end{aligned}
$$

In the momentum-space representation and with equal time components, $u_{1}=u_{2}$ we have

$$
\phi_{\boldsymbol{Q} ; \sigma_{1}, \sigma_{2}}\left(\boldsymbol{r}_{i_{1}}-\boldsymbol{r}_{i_{2}} ; 0\right)=\frac{1}{N} \sum_{\boldsymbol{k}} e^{i k \cdot\left(\boldsymbol{r}_{i_{1}}-\boldsymbol{r}_{i_{2}}\right)} \phi_{\sigma_{1}, \sigma_{2}}(\boldsymbol{k}, \boldsymbol{Q})
$$

It is widely accepted that the generalized random phase is a good approximation for the collective excitations in a weak-coupling regime, and therefore, it can 
be used to separate the solutions of the Dyson and the Bethe-Salpeter equations. In this approximation, the single-particle excitations are replaced with those obtained by diagonalizing the Hartree-Fock (HF) Hamiltonian; while the collective modes are obtained by solv- ing the BS equation in which the single-particle Green's functions are calculated in $\mathrm{HF}$ approximation, and the BS kernel is obtained by summing ladder and bubble diagrams.

Inserting expansion (24) in Eq. (21) using (23)

$$
\phi_{\boldsymbol{q}, \sigma_{1}, \sigma_{2}}(\boldsymbol{k}, \boldsymbol{Q})=\sum_{\sigma_{3}, \sigma_{4}, \sigma_{1}^{\prime}, \sigma_{2}^{\prime}} \sum_{\imath \omega_{m}} G_{\boldsymbol{q}}^{\sigma_{1} \sigma_{3}}\left(\boldsymbol{k}+\boldsymbol{Q}, \imath \omega_{m}+\omega(\boldsymbol{Q})\right) G_{\boldsymbol{q}}^{\sigma_{4} \sigma_{2}}\left(\boldsymbol{k}, \imath \omega_{m}\right) I_{\sigma_{3}, \sigma_{4}, \sigma_{1}^{\prime}, \sigma_{2}^{\prime}} \sum_{\boldsymbol{p}} \phi_{\boldsymbol{q}, \sigma_{1}^{\prime}, \sigma_{2}^{\prime}}(\boldsymbol{p}, \boldsymbol{Q}),
$$

where the kernel represents the direct and exchange interactions:

$$
\begin{aligned}
I_{\sigma_{1}, \sigma_{2}, \sigma_{3}, \sigma_{4}} & =I_{\sigma_{1}, \sigma_{2}, \sigma_{3}, \sigma_{4}}^{d}+I_{\sigma_{1}, \sigma_{2}, \sigma_{3}, \sigma_{4}}^{\text {exch }}, \\
I_{\sigma_{1}, \sigma_{2}, \sigma_{3}, \sigma_{4}}^{d} & =-U \delta_{\sigma_{1}, \sigma_{3}} \delta_{\sigma_{2}, \sigma_{4}}, \\
I_{\sigma_{1}, \sigma_{2}, \sigma_{3}, \sigma_{4}}^{e x c h} & =U \delta_{\sigma_{1}, \sigma_{2}} \delta_{\sigma_{3}, \sigma_{4}}
\end{aligned}
$$

We now introduce the compact notation $\hat{\phi}_{\boldsymbol{q}}(\boldsymbol{k}, \boldsymbol{Q})=$ $\left[\phi_{\boldsymbol{q}, \downarrow, \uparrow}(\boldsymbol{k}, \boldsymbol{Q}), \phi_{\boldsymbol{q}, \uparrow, \downarrow}(\boldsymbol{k}, \boldsymbol{Q}), \phi_{\boldsymbol{q}, \uparrow, \uparrow}(\boldsymbol{k}, \boldsymbol{Q}), \phi_{\boldsymbol{q}, \downarrow, \downarrow}(\boldsymbol{k}, \boldsymbol{Q})\right]^{T}$ (where $T$ means the transpose vector). Then, the equation for the BS amplitudes becomes

$$
\hat{\phi}_{\boldsymbol{q}}(\boldsymbol{k}, \boldsymbol{Q})=-U \hat{D} \sum_{\mathbf{p}} \hat{\phi}_{\boldsymbol{q}}(\mathbf{p}, \boldsymbol{Q})+U \hat{M} \sum_{\mathbf{p}} \hat{\phi}_{\boldsymbol{q}}(\mathbf{p}, \boldsymbol{Q})
$$

Here, $U \widehat{D}$ and $U \widehat{M}$ represent the direct and exchange interactions, respectively:

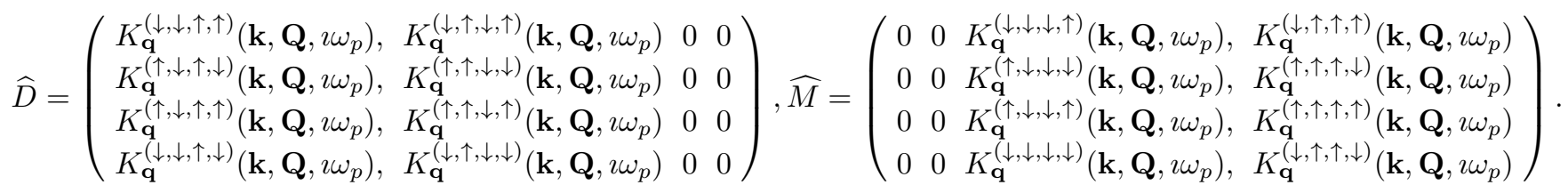

Here, $\omega_{p}=(2 \pi / \beta) p ; p=0, \pm 1, \pm 2, \ldots$ is a Bose frequency, and we have introduced the two-particle propagator $K_{\mathbf{q}}^{(i, j, k, l)}\left(\mathbf{k}, \mathbf{Q}, \imath \omega_{p}\right)=\sum_{\omega_{m}} G_{\mathbf{q}}^{i, j}\left(\mathbf{k}+\mathbf{Q} ; \imath \omega_{p}+\imath \omega_{m}\right) G_{\mathbf{q}}^{k, l}\left(\mathbf{k} ; \imath \omega_{m}\right)$, where $i, j, k, l=\{\uparrow, \downarrow\}$. The condition for existing a non-trivial solution of the Bethe-Salpeter equations leads to the following secular determinant

$$
Z=\left|\begin{array}{cccc}
U^{-1}+\left(I_{\gamma, \gamma}-L_{\tilde{\gamma}, \widetilde{\gamma}}\right) & \left(J_{\gamma, l}-K_{m, \widetilde{\gamma}}\right) & \left(I_{\gamma, \widetilde{\gamma}}+L_{\gamma, \widetilde{\gamma}}\right) & \left(J_{\gamma, m}+K_{l, \tilde{\gamma}}\right) \\
\left(J_{\gamma, l}-K_{m, \tilde{\gamma}}\right) & U^{-1}+\left(I_{l, l}-L_{m, m}\right) & \left(J_{l, \tilde{\gamma}}+K_{m, \gamma}\right) & \left(I_{l, m}+L_{l, m}\right) \\
\left(I_{\gamma, \tilde{\gamma}}+L_{\gamma, \widetilde{\gamma}}\right) & \left(J_{l, \tilde{\gamma}}+K_{m, \gamma}\right) & -U^{-1}+\left(I_{\widetilde{\gamma}, \widetilde{\gamma}}-L_{\gamma, \gamma}\right) & \left(J_{\tilde{\gamma}, m}-K_{\gamma, l}\right) \\
\left(J_{\gamma, m}+K_{l, \widetilde{\gamma}}\right) & \left(I_{l, m}+L_{l, m}\right) & \left(J_{\widetilde{\gamma}, m}-K_{\gamma, l}\right) & U^{-1}+\left(I_{m, m}-L_{l, l}\right)
\end{array}\right|,
$$

where the following symbols are used:

$$
\begin{gathered}
I_{a, b}=\frac{1}{2 N} \sum_{\mathbf{k}} a_{\mathbf{k}, \mathbf{Q}}^{\mathbf{q}} b_{\mathbf{k}, \mathbf{Q}}^{\mathbf{q}}\left[\frac{1-f\left(\omega_{-}(\mathbf{k}, \mathbf{q})\right)-f\left(\omega_{+}(\mathbf{k}+\mathbf{Q}, \mathbf{q})\right)}{\left.\omega+\Omega_{\mathbf{q}}(\mathbf{k}, \mathbf{Q})-\varepsilon_{\mathbf{q}}(\mathbf{k}, \mathbf{Q})\right]}-\frac{1-f\left(\omega_{+}(\mathbf{k}, \mathbf{q})\right)-f\left(\omega_{-}(\mathbf{k}+\mathbf{Q}, \mathbf{q})\right)}{\left.\left.\omega+\Omega_{\mathbf{q}} \mathbf{k}, \mathbf{Q}\right) \varepsilon_{\mathbf{q}}(\mathbf{k}, \mathbf{Q})\right]}\right], \\
J_{a, b}=\frac{1}{2 N} \sum_{\mathbf{k}} a_{\mathbf{k}, \mathbf{Q}}^{\mathbf{q}} b_{\mathbf{k}, \mathbf{Q}}^{\mathbf{q}}\left[\frac{1-f\left(\omega_{-}(\mathbf{k}, \mathbf{q})\right)-f\left(\omega_{+}(\mathbf{k}+\mathbf{Q}, \mathbf{q})\right)}{\left.\omega+\Omega_{\mathbf{q}}(\mathbf{k}, \mathbf{Q})-\varepsilon_{\mathbf{q}}(\mathbf{k}, \mathbf{Q})\right]}+\frac{1-f\left(\omega_{+}(\mathbf{k}, \mathbf{q})\right)-f\left(\omega_{-}(\mathbf{k}+\mathbf{Q}, \mathbf{q})\right)}{\left.\omega+\Omega_{\mathbf{q}}(\mathbf{k}, \mathbf{Q})+\varepsilon_{\mathbf{q}}(\mathbf{k}, \mathbf{Q})\right]}\right], \\
K_{a, b}=\frac{1}{2 N} \sum_{\mathbf{k}} a_{\mathbf{k}, \mathbf{Q}}^{\mathbf{q}} b_{\mathbf{k}, \mathbf{Q}}^{\mathbf{q}}\left[\frac{f\left(\omega_{-}(\mathbf{k}, \mathbf{q})\right)-f\left(\omega_{-}(\mathbf{k}+\mathbf{Q}, \mathbf{q})\right)}{\left.\omega+\Omega_{\mathbf{q}}(\mathbf{k}, \mathbf{Q})+\epsilon_{\mathbf{q}}(\mathbf{k}, \mathbf{Q})\right]}+\frac{f\left(\omega_{+}(\mathbf{k}, \mathbf{q})\right)-f\left(\omega_{+}(\mathbf{k}+\mathbf{Q}, \mathbf{q})\right)}{\left.\omega+\Omega_{\mathbf{q}}(\mathbf{k}, \mathbf{Q})-\epsilon_{\mathbf{q}}(\mathbf{k}, \mathbf{Q})\right]}\right], \\
L_{a, b}=\frac{1}{2 N} \sum_{\mathbf{k}} a_{\mathbf{k}, \mathbf{Q}}^{\mathbf{q}} b_{\mathbf{k}, \mathbf{Q}}^{\mathbf{q}}\left[\frac{f\left(\omega_{-}(\mathbf{k}, \mathbf{q})-f\left(\omega_{-}(\mathbf{k}+\mathbf{Q}, \mathbf{q})\right)\right.}{\left.\omega+\Omega_{\mathbf{q}}(\mathbf{k}, \mathbf{Q})+\epsilon_{\mathbf{q}}(\mathbf{k}, \mathbf{Q})\right]}-\frac{f\left(\omega_{+}(\mathbf{k}, \mathbf{q})\right)-f\left(\omega_{+}(\mathbf{k}+\mathbf{Q}, \mathbf{q})\right)}{\left.\omega+\Omega_{\mathbf{q}}(\mathbf{k}, \mathbf{Q})-\epsilon_{\mathbf{q}}(\mathbf{k}, \mathbf{Q})\right]}\right] .
\end{gathered}
$$

Here, $\varepsilon_{\boldsymbol{q}}(\boldsymbol{k}, \boldsymbol{Q})=E_{\boldsymbol{q}}(\boldsymbol{k}+\boldsymbol{Q})+E_{\boldsymbol{q}}(\boldsymbol{k}), \epsilon_{\boldsymbol{q}}(\boldsymbol{k}, \boldsymbol{Q})=E_{\boldsymbol{q}}(\boldsymbol{k}+$ $\boldsymbol{Q})-E_{\boldsymbol{q}}(\boldsymbol{k}), \Omega_{\boldsymbol{q}}(\boldsymbol{k}, \boldsymbol{Q})=\eta_{\boldsymbol{q}}(\boldsymbol{k})-\eta_{\boldsymbol{q}}(\boldsymbol{k}+\boldsymbol{Q})$, and $a$ and $b$ are one of the following form factors:

$$
\begin{aligned}
& \gamma_{\boldsymbol{k}, \boldsymbol{Q}}^{\boldsymbol{q}}=u_{\boldsymbol{k}}^{\boldsymbol{q}} u_{\boldsymbol{k}+\boldsymbol{Q}}^{\boldsymbol{q}}+v_{\boldsymbol{k}}^{\boldsymbol{q}} v_{\boldsymbol{k}+\boldsymbol{Q}}^{\boldsymbol{q}}, \\
& l_{\boldsymbol{k}, \boldsymbol{Q}}^{\boldsymbol{q}}=u_{\boldsymbol{k}}^{q} u_{\boldsymbol{k}+\boldsymbol{Q}}^{\boldsymbol{q}}-v_{\boldsymbol{k}}^{\boldsymbol{q}} v_{\boldsymbol{k}+\boldsymbol{Q}}^{\boldsymbol{q}}, \\
& \widetilde{\gamma}_{\boldsymbol{k}, \boldsymbol{Q}}^{\boldsymbol{q}}=u_{\boldsymbol{k}}^{\boldsymbol{q}} v_{\boldsymbol{k}+\boldsymbol{Q}}^{\boldsymbol{q}}-u_{\boldsymbol{k}+\boldsymbol{Q}}^{\boldsymbol{q}} v_{\boldsymbol{k}}^{\boldsymbol{q}}, \\
& m_{\boldsymbol{k}, \boldsymbol{Q}}^{\boldsymbol{q}}=u_{\boldsymbol{k}}^{q} v_{\boldsymbol{k}+\boldsymbol{Q}}^{q}+u_{\boldsymbol{k}+\boldsymbol{Q}}^{q} v_{\boldsymbol{k}}^{q} \text {. }
\end{aligned}
$$


According to the well-known Goldstone theorem, as $\boldsymbol{Q} \rightarrow$ 0 , there exists a solution $\omega \rightarrow 0$. In this limit all $J, K$ and $L$ vanish, and the secular equation reduces to the gap equation written as $0=1+U I_{\gamma=1, \gamma=1}$.

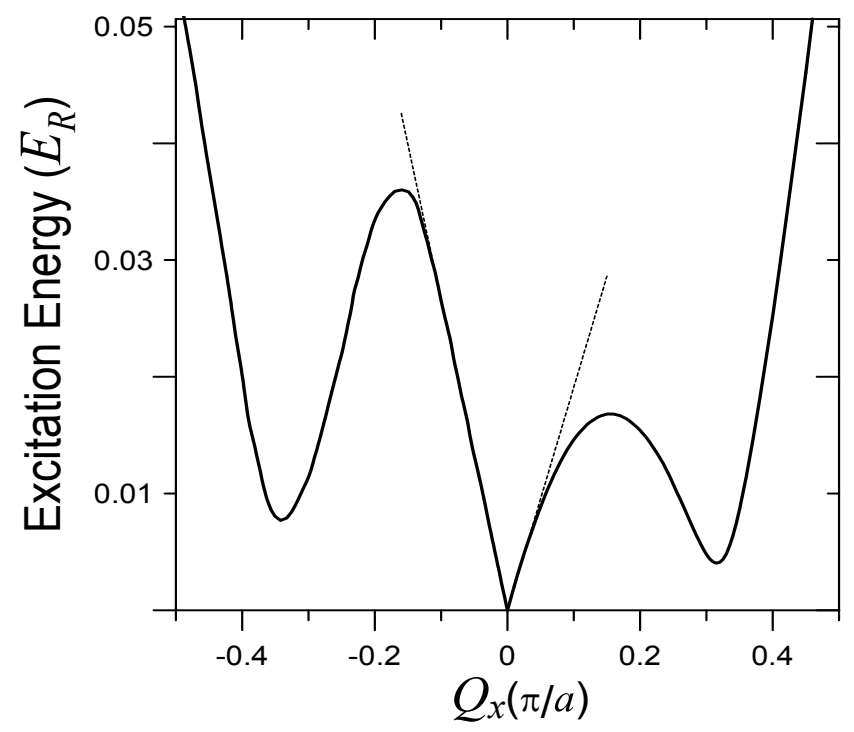

FIG. 5: Excitation energy for collective modes of a polarized ${ }^{6} \mathrm{Li}$ gas in a 3D optical lattice with $\lambda=1030 \mathrm{~nm}$ and total filling factor $f=0.4685$. The Hubbard parameters are $J=$ $0.078 E_{R}$ and the attractive on-site interaction is $U / J=2.64$.

For $\boldsymbol{Q} \neq 0$, we use a 3D Gaussian integration in each term in the secular determinant (27) and search for the solution when $Z=0$. Without loss of generality, we fix the collective excitation momentum $\boldsymbol{Q}$ in the $x$-direction, $\left(Q_{x}, 0,0\right)$. For small values of $Q_{x}$ the excitation energy is the linear, low-energy (Goldstone) mode in the FFLO state corresponding to the fluctuations of the order parameter phase, but since the FFLO state breaks both gauge and translational symmetry there are two distinct modes as shown in Fig. 5. In this case, the polarization is $P=0.093883$; the filling-fraction parameters are $f_{\uparrow}=0.256248$ and $f_{\downarrow}=0.212263$, and $U / J=2.64$ at a temperature $k_{B} T / E_{R}=10^{-4}$. The two distinct sound velocities in the long wavelength limit are $8.56 \mathrm{~mm} / \mathrm{s}$ and $6.14 \mathrm{~mm} / \mathrm{s}$ as shown for the negative and positive wavenumbers, respectively. The results from our numerical solutions of the BS equation also show that the Goldstone modes have rotonlike minima, $\omega_{r}=0.0077 E_{R}$ and $\omega_{r}=0.004 E_{R}$.

In Fig. [5 the rotonlike structure is clearly seen and the minimum requirements on the flow velocities to be able to slow down (obtained from the two roton slopes) are $v_{1}=0.725 \mathrm{~mm} / \mathrm{s}$ and $v_{2}=0.41 \mathrm{~mm} / \mathrm{s}$, respectively. The asymmetry of the sound mode and the roton minima originates from the fact that the population imbalance is achieved when either $\omega_{+}\left(\boldsymbol{k}+\boldsymbol{Q}, q_{x}\right)$ or $\omega_{-}\left(\boldsymbol{k}+\boldsymbol{Q}, q_{x}\right)$ is negative in some regions of momentum space, but the regions are different for positive and negative $Q_{x}$. The answer of the question how this asymmetry is related to $f_{\uparrow}, f_{\downarrow}$ and $U / J$ requires analytical expressions for the two regions which is beyond the goals of the present work.

\section{CONCLUSIONS}

In this paper we have presented the phase diagram and the collective excitations of an imbalanced system of ${ }^{6} \mathrm{Li}$ atoms loaded in a cubic optical lattice. Upon minimization of the free energy, the stability regions of BCS, Sarma, FFLO and BCS-normal mixed-state phases were obtained. We also showed that the FFLO phase can be quite large compared to both, the Sarma and the phase separation regions when the hopping strength in the single-band Hubbard model is increased and the onsite attraction is decreased. The effects of dimensionality were also analyzed by contrasting the phases of a system loaded in a 3D optical lattice with an identical, 2D system where we showed that the lower dimensionality gas can sustain larger polarizations in the FFLO phase.

We also derived a Bethe-Salpeter equation for the attractive Hubbard Hamiltonian based on the generalized random phase approximation to calculate the collective mode spectrum of the Fermi gas in a deep optical lattice. Using a contact interaction, an algebraic equation for the BS amplitudes was obtained. The solution for the excitation spectrum of collective modes was derived by calculating the roots of the corresponding secular $4 \times 4$ determinant. For $\boldsymbol{Q} \rightarrow 0$ we obtained two distinct Goldstone modes and their respective sound velocities. For shorter wavelengths, we showed that the Goldstone modes have an asymmetric rotonlike spectrum. The critical flow velocities in this region were calculated to show that superfluidity can survive in a polarized fermion gas in twoand in three-dimensional optical lattices.

This work was partially supported by UNAM-DGAPA grants IN-105011 \& IN-111613, and Conacyt 104917.
1 M. Greiner, O. Mandel, T. Esslinger, T.W. Hänsch and I. Bloch, Nature 415, 39 (2002).

2 T. Esslinger, Ann. Rev. Condensed Matter Phys. 1, 129 (2010).

3 I. Bloch, J. Dalibard, and W. Zwerger, Rev. Mod. Phys.
80, 885 (2008); J. P. A. Devreese, S. Klimin, M. Wouters, and J. Tempere, Modern Phys. Lett. B, 26, 1230014 (2012)

4 W. Ketterle, Y. Shin, A. Schirotzek and C. H. Schunk, J. Phys, Condensed Matter 21, 164206 (2009).

5 Yean-an Liao, A.S.C. Rittner, T. Paprotta, W. Li, G.B. 
Partridge, R.G. Hulet, S.K. Baur and E.J. Mueller, Nature 467, 567 (2010).

${ }^{6}$ P. Fulde, and R. A. Ferrell, Phys. Rev. 135, A550 (1964).

7 A. I. Larkin, and Y. N. Ovchinnikov, Zh. Eksp. Teor. Fiz., 47, 1136 (1964) [Sov. Phys. JETP 20, 762 (1965)].

8 P. Pieri, D. Neilson, and G. C. Strinati, Phys. Rev. B 75, 113301 (2007); T. Hakioğlu and M. Şahin, Phys. Rev. Lett. 98, 166405 (2007); T. Zhou and C. S. Ting, Phys. Rev. B 80, 224515 (2009); Xian-Jun Zuo and Chang-De Gong, Eur. Phys. Lett. 86, 47004 (2009); H. Shimahara Phys. Rev. B 80, 214512 (2009); A. Romano et al., Phys. Rev. B 81, 064513 (2010); R. Ikeda, Phys. Rev. B 81, 060510(R) (2010); M. M. Maśka et al., Phys. Rev. B 82, 054509 (2010).

9 T. Koponen et al., New Journal of Physics 8, 179 (2006)

10 T. Koponen et al., Phys. Rev. Lett. 99, 120403 (2007); T. Paananen, T. K. Koponen, P. Törma, and J.P. Martikainen, Phys. Rev. A 77, 053602, (2008).

11 Tung-Lam Dao, A. Georges, and M. Capone, Phys. Rev. B 76, 104517 (2007); Q. Chen et al., Phys. Rev. B 75, 014521 (2007); Xia-Ji Liu, H. Hu, and P. D. Drummond, Phys. Rev. A 76, 043605 (2007); M. Rizzi, et al., Phys. Rev. B 77, 245105 (2008); Xia-Ji Liu, Hui Hu, and P. D. Drummond, Phys. Rev. A 78, 023601 (2008); M. Reza Bakhtiari, M. J. Leskinen, and P. Törma, Phys. Rev. Lett. 101, 120404 (2008); A. Lazarides and B. Van Schaeybroec, Phys. Rev. A 77, 041602 (2008); T Paananen, J. Phys. B: At. Mol. Opt. Phys. 42165304 (2009); X. Cui and Y. Wang, Phys. Rev. B 79, 180509(R) (2009); A. Mishra and H. Mishra, Eur. Phys. J. D 53, 75 (2009); B. Wang, Han-Dong Chen, and S. Das Sarma, Phys. Rev. A 79, 051604(R) (2009); Y. Yanase, Phys. Rev. B 80, 220510(R) (2009); A. Ptok, M. Máska, and M. Mierzejewski, J. Phys.:
Condens. Matter 21, 295601 (2009); Yan Chen et al., Phys. Rev. B 79, 054512 (2009); Yen Lee Loh and N. Trivedi, Phys. Rev. Lett. 104, 165302 (2010); A. Korolyuk, F. Massel, and P. Törma, Phys. Rev. Lett. 104, 236402 (2010); F. Heidrich-Meisner et al., Phys. Rev. A 81, 023629 (2010); S. K. Baur, J. Shumway, and E. J. Mueller, Phys. Rev. A 81, 033628 (2010); A. Korolyuk, F. Massel, and P. Törmä, Phys. Rev. Lett. 104, 236402 (2010); M. J. Wolak et al., Phys. Rev. A 82, 013614 (2010); L. Radzihovsky and D. Sheehy, Rep. Prog. Phys. 73, (2010) 076501

12 J. M. Edge and N. R. Cooper, Phys. Rev. Lett. 103, 065301 (2009); Phys. Rev. A 81, 063606 (2010).

13 A. Sedrakian and D. H. Rischke, Phys. Rev. D 80, 074022 (2009).

14 G. Sarma, J. Phys. Chem. 24, 1029 (1963).

15 Z. G. Koinov, R. Mendoza and M. Fortes, Phys. Rev. Lett. 106, 100402 (2011).

16 Y. Shin, C. H. Schunck, A. Schirotzek, and W. Ketterle, Nature 451, 689 (2008);

17 P. F. Bedaque, H. Caldas, and G. Kupak, Phys. Rev. Lett. 91, 247002 (2003); H. Caldas, Phys Rev. A 69, 063602 (2004); H. Caldas, C. W. Morais and A. L. Mota, Phys. Rev. D 72, 045008 (2005); S. Sachdev and K. Yang, Phys. Rev. B 73, 174504 (2006).

18 L. Belkhir and M. Randeria, Phys. Rev. B 49, 6829 (1994).

19 R. Cotê and A. Griffin, Phys. Rev. B 48, 10404 (1993).

20 Z. G. Koinov, Physica C 407, 470 (2010); Physica Status Solidi (B) 247, 140 (2010); Ann. Phys. (Berlin) 522, 693 (2010); cond-mat/1010.1200.

21 K. V. Samokhin, Phys. Rev. B 81, 224507 (2010).

22 Y.-P. Shim, R. A. Duine, and A. H. MacDonald, Phys. Rev. A 74, 053602 (2006). 